Effective heat conduction in a configuration with nonoverlapped magnetic islands

A. Gupta, and M. Z. Tokar

Citation: Physics of Plasmas 15, 034503 (2008); doi: 10.1063/1.2896576

View online: https://doi.org/10.1063/1.2896576

View Table of Contents: http://aip.scitation.org/toc/php/15/3

Published by the American Institute of Physics

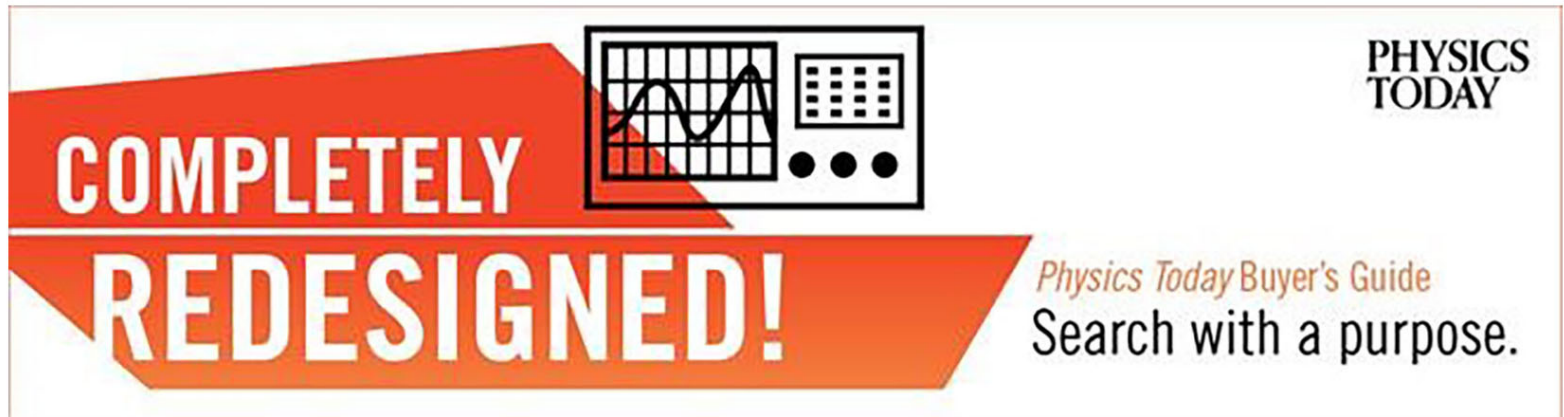




\title{
Effective heat conduction in a configuration with nonoverlapped magnetic islands
}

\author{
A. Gupta and M. Z. Tokar \\ Institut für Energieforschung - Plasmaphysik, Forschungszentrum Jülich, Association EURATOM-FZJ, \\ Trilateral Euregio Cluster, Jülich, Germany
}

(Received 17 December 2007; accepted 22 February 2008; published online 25 March 2008)

\begin{abstract}
The effective radial heat conduction $\kappa_{\text {eff }}$ in a plasma configuration with nonoverlapped magnetic island chains is assessed by applying an "optimal path" method. This approach implies that heat is transported predominantly along paths rendering the minimum temperature variation and is related to the principle of minimum entropy production. Paths combined of up to three radial sections and two segments aligned along magnetic field lines are considered. It is demonstrated that the enhancement of $\kappa_{\text {eff }}$ over the level of perpendicular heat conduction $\kappa_{\perp}$ arising due to flows along magnetic field lines is controlled only by the Chirikov parameter and by the value $4 b_{r}^{2} \kappa_{\|} / \kappa_{\perp}$, where $b_{r}$ is the relative amplitude of the radial field resonant harmonic and $\kappa_{\|}$is the parallel heat conduction. (C) 2008 American Institute of Physics. [DOI: 10.1063/1.2896576]
\end{abstract}

Magnetic islands are a well known phenomena in hot fusion plasmas generated by resonant perturbations of the magnetic field. The latter arise from different sources such as MHD instabilities developing spontaneously when some parameters approach a critical level, e.g., neoclassical tearing modes, ${ }^{1}$ or special coils introduced in order to control the plasma behavior. $^{2-5}$ Recently such coils have been successfully used for mitigation or even complete suppression of the edge localized modes in DIII-D (Refs. 6 and 7) and JET (Ref. 8) tokamaks.

The magnetic field perturbations can be represented as a series of Fourier harmonics,

$$
\widetilde{\mathbf{B}}=\sum_{M, N} \widetilde{\mathbf{B}}_{M, N} \sin \psi
$$

where $\psi=M \vartheta-N \varphi$ is the harmonic phase with integer multiplicities $M$ and $N$ in the poloidal and toroidal directions $\vartheta$ and $\varphi$, respectively. It is essential that such perturbations have radial components $\widetilde{B}_{M, N}^{r}$ which are not present in the main tokamak field composed of the poloidal and toroidal components $B_{\vartheta}$ and $B_{\varphi}$, respectively. Due to $\widetilde{B}_{M, N}^{r}$ field lines deviate in the radial direction. This is especially pronounced in the vicinity of the so called resonant magnetic surfaces (RS) where the safety factor $q \equiv B_{\varphi} r /\left(B_{\vartheta} R\right)$, with $r$ and $R$ are the minor and major radii of the surface, is equal to the ratio $M / N$. Here the corresponding Fourier harmonic is constant along field lines. However due to nonzero magnetic shear $\hat{s}=r / q \times d q / d r$ the resonance with the perturbation weakens with the distance from the RS.

By integrating the line of force equation $d \mathbf{l} \times \mathbf{B}=0$, one can demonstrate that in the plane $r, \psi$ the behavior of perturbed field lines is governed by the equation, ${ }^{10}$

$$
y^{2}-\frac{\cos \psi}{2}=C
$$

Here $y$ is the deviation from the RS measured in $\delta=2 \sqrt{b_{r} R r_{0} /(\hat{s} N)}$, with $b_{r}=\widetilde{B}_{M, N}^{r} / B_{\varphi} ; \delta$ is the maximum dis- tance between RS and the surface described by Eq. (2) with $C=1 / 2$. The latter is called separatrix since it separates the regions of qualitatively different behavior. For $C>1 / 2$ the perturbation do not change the topology of original magnetic surfaces and waves them slightly in the radial direction; for $C<1 / 2$ the topology is changed so that magnetic surfaces encircle the short-circuited field line passing through the point $\psi=0$ on the RS. The cross section of the separatrix by the plane $r, \psi$ looks like a chain of $M$ magnetic islands with the radial width $w=2 \delta$, see Fig. 1 .

The deviation of lines of force from unperturbed magnetic surfaces results in an enhancement of the effective particle and heat losses from the plasma; radial gradients of plasma parameters have nonzero projections along such lines and drive parallel particle and heat flows which contribute to the normal perpendicular transport. In this paper we propose a model in order to assess the effective radial heat conduction in a configuration where the heat flux from the plasma core comes through nonoverlapped island chains. On the one hand, compared to previous analytical studies ${ }^{9,10}$ the present approach treats on the same foot cases with arbitrary parameter $\varsigma=4 \kappa_{\|} b_{r}^{2} / \kappa_{\perp}$, where $\kappa_{\perp}$ and $\kappa_{\|}$are the heat conduction components perpendicular and parallel to the magnetic field. The parameter $s$ is equal to $\left(w / w_{c}\right)^{4}$ with $w_{c}$ being the critical island width introduced in Ref. 10. There the limit cases of "small," $w \ll w_{c}(\mathbf{1} \ll 1)$, and "large," $w \gg w_{c}(\mathrm{~s} \gg 1)$, islands have been considered analytically. On the other hand, it illustrates in a more transparent way than a pure numerical approach $^{11,12}$ what parameters are of concern for the effective transport characteristics, and provides a computationally economical method to find the dependencies on this parameters.

The "optimal path" method applied below was used in Ref. 13 for assessment of transport characteristics in a stochastic magnetic field. It asserts that in a region where heat sources are negligible, in particular, at the plasma edge, the heat supplied from the plasma core is transferred predominantly along paths providing the minimum temperature 

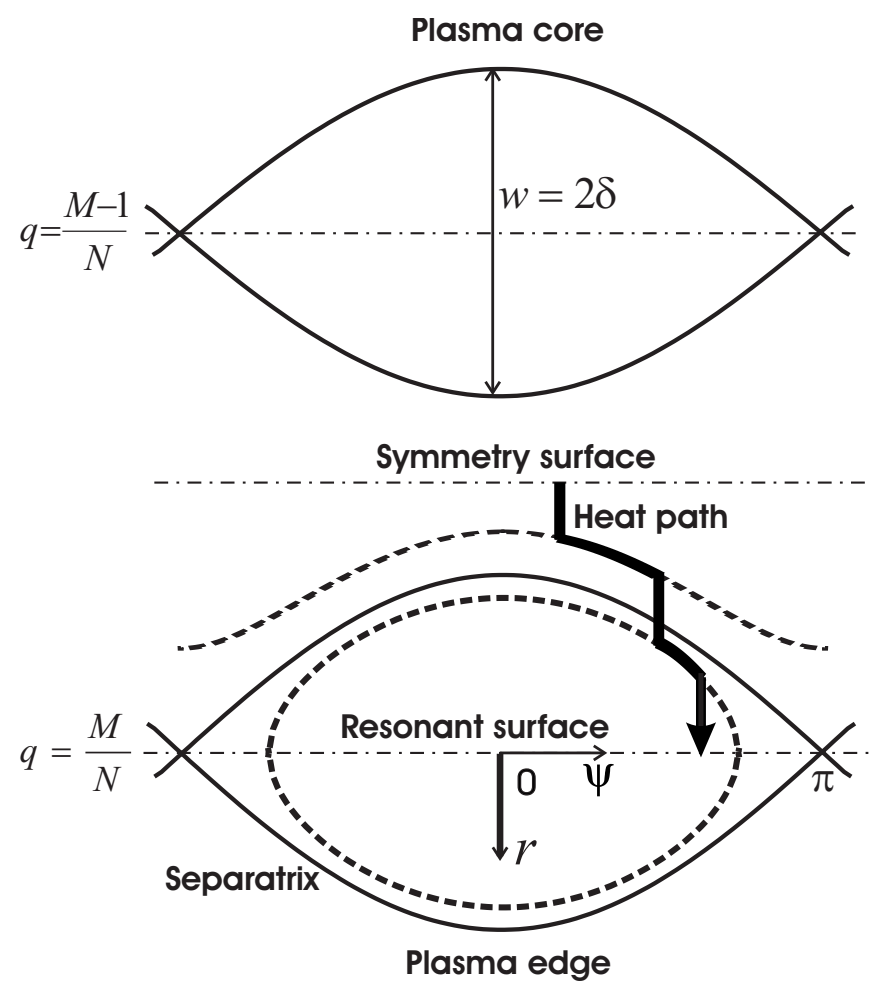

FIG. 1. Schematic view of nonoverlapped magnetic island chains with an elementary section of a heat path.

change. There is a direct relation between this approach and the principle of minimum entropy production. ${ }^{14}$ Under conditions in question, i.e., for zero heat source, the entropy production rate per unit volume, $\theta$, is given by the relation $\theta=\boldsymbol{\Gamma} \cdot \boldsymbol{\nabla}(1 / T),{ }^{15}$ where $\boldsymbol{\Gamma}$ is the heat flux density. By applying this relation to a toroidal shell between two close radial positions, $r_{1,2}$, so that $r_{2}-r_{1} \ll r_{1,2}$, one gets for the total entropy production rate in this shell,

$$
\int \theta d V=\frac{P \Delta T}{T_{1} T_{2}}
$$

with $P$ being the total power transferred through the shell, $T_{1,2}$ the averaged temperatures on its boundary surfaces and $\Delta T=T_{1}-T_{2}$. Thus, the minimum entropy production rate accords to the minimum temperature change $\Delta T$.

Henceforth we consider the case of $M \gg 1$ so that $b_{r}$ is not very different for the adjacent RS and the islands have close dimensions in the radial and $\psi$ directions. All heat paths can be combined from elementary sections connecting the symmetry plane and the RS of one of the island chains, see Fig. 1. The dimensionless distance between these two surfaces, $y_{0}$, is equal to $1 / \sigma_{\mathrm{Ch}}$ where $\sigma_{\mathrm{Ch}}$ is the so-called Chirikov parameter. ${ }^{4}$ The situation with nonoverlapped island chains considered here corresponds to $\sigma_{\mathrm{Ch}}<1$. Henceforth we take into account only heat paths which are composed from sections aligned either in the radial direction or are parallel to the magnetic field. In the former case the radial flux with the density $\Gamma_{r}$ is mostly transported by the perpendicular heat conduction,

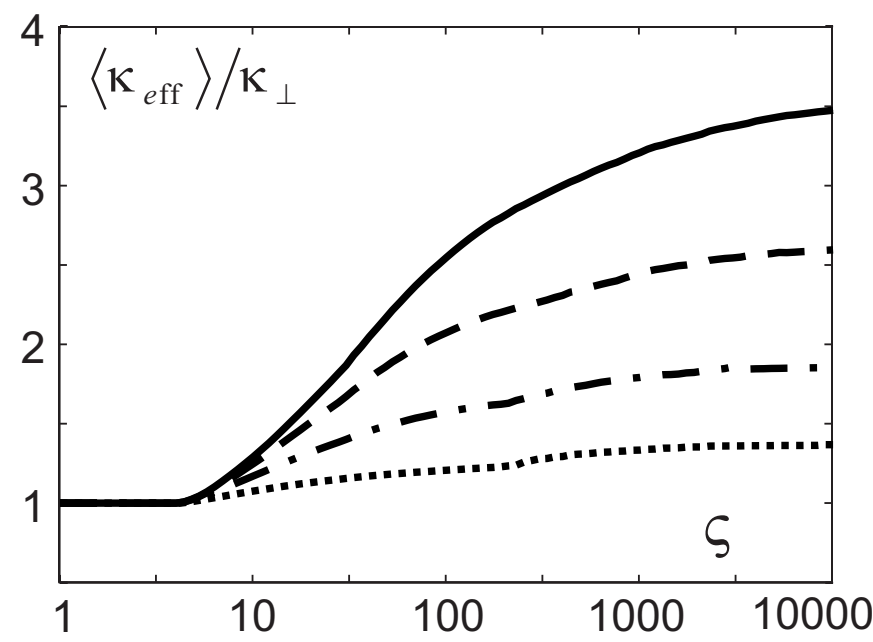

FIG. 2. The ratio of the effective radial heat conduction to the perpendicular one as a function of the parameter $\varsigma=4 \kappa_{\|} b_{r}^{2} / \kappa_{\perp}$ computed for different magnitudes of the Chirikov parameter $\sigma_{\mathrm{Ch}}: \sigma_{\mathrm{Ch}}=0.9$ (solid curve), 0.8 (dashed curve), 0.6 (dashed-dotted curve), and 0.3 (dotted curve).

$$
\Gamma_{r} \approx-\kappa_{\perp} \partial T / \partial r
$$

and in the latter one, by the parallel one,

$$
\Gamma_{r} \approx-\left(\kappa_{\|} \nabla_{\|} T\right) B_{r} / B=-\kappa_{\|} b_{r}^{2} \sin ^{2} \psi \partial T / \partial r .
$$

We consider paths including one or two parallel sections which can be placed either inside or outside the island, see Fig. 1. The initial and final positions of these section are varied in the whole space from the symmetry surface to the RS and optimal paths with the minimum total temperature change are selected. The selection procedure does not exclude sections of zero length. Therefore, depending on the perturbation strength and initial phase of the path at the symmetry surface, the optimal one can be a combination of up to two parallel and three radial segments. The approach can be straightforwardly generalized on a larger number of parallel sections. This leads to a noticeable increase of computation time but, presumably, does not provide any significant improvement, as it follows from comparison of results obtained for paths with one and two parallel segments.

The total temperature change along all radial sections of such a heat path follows from integration of Eqs. (4). With constant $\kappa_{\perp}$ one gets

$$
\Delta_{\perp} T=\frac{\Gamma_{r} w}{2 \kappa_{\perp}}\left[y_{0}-\sum_{j=1}^{j_{\max }}\left(y_{b}^{j}-y_{t}^{j}\right)\right],
$$

where $y_{b, t}^{j}$ are the distances from the RS of the beginning and terminating points of the parallel section $j$ and $j_{\max }=1$ or 2 in this study. By using the relation (2) we get from Eq. (5) for the parallel path sections,

$$
\Delta_{\|}^{j} T=\frac{\Gamma_{r} w}{8 \kappa_{\|} b_{r}^{2}} \eta_{j}
$$

with 


\section{Symmetry surface}
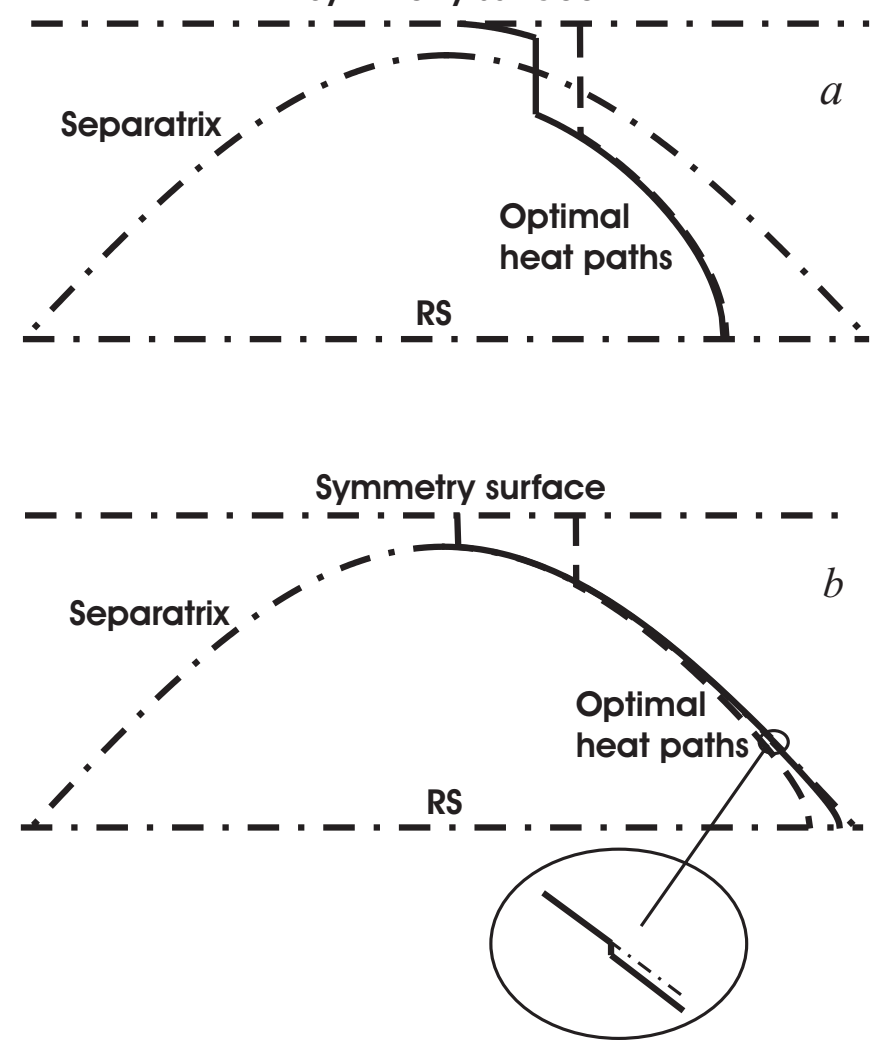

FIG. 3. Optimal paths beginning at the symmetry surface with the initial phases 0.1 (solid curves) and 1 (dashed curves) found for $\mathrm{s}=10$ (a) and $\mathrm{s}=10^{4}(\mathrm{~b})$ with $\sigma_{\mathrm{Ch}}=0.9$.

$$
\eta_{j}=\frac{1}{2 a} \ln \frac{\left(a+y_{b}^{j}\right)\left(a-y_{t}^{j}\right)}{\left(a+y_{t}^{j}\right)\left(a-y_{b}^{j}\right)}+\frac{1}{2 c} \ln \frac{\left(y_{b}^{j}-c\right)\left(y_{t}^{j}+c\right)}{\left(y_{t}^{j}-c\right)\left(y_{b}^{j}+c\right)}
$$

for a section inside the island and

$$
\eta_{j}=\frac{1}{2 a} \ln \frac{\left(a+y_{b}^{j}\right)\left(a-y_{t}^{j}\right)}{\left(a+y_{t}^{j}\right)\left(a-y_{b}^{j}\right)}+\frac{1}{c}\left(\arctan \frac{y_{b}^{j}}{c}-\arctan \frac{y_{t}^{j}}{c}\right)
$$

for that outside.

Here

$$
\begin{aligned}
& a=\sqrt{\frac{1-\cos \psi_{j}}{2}+\left(y_{b}^{j}\right)^{2}} \\
& c=\sqrt{\frac{1+\cos \psi_{j}}{2}-\left(y_{b}^{j}\right)^{2} \mid},
\end{aligned}
$$

where $\psi_{j}$ is the initial phase of the $j$ path parallel section; the initial phases of consequent sections are related as follows:

$$
\cos \psi_{j+1}=\cos \psi_{j}+2\left[\left(y_{t}^{j}\right)^{2}-\left(y_{b}^{j}\right)^{2}\right] .
$$

Optimal paths are selected by varying $y_{b, t}^{j=1, \ldots, j_{\max }}$ in the whole range $\left[0, y_{0}\right]$ and looking for the minimal total temperature change $\Delta T=\Delta_{\perp} T+\sum_{j=1}^{j_{\max }} \Delta_{\|}^{j} T$. The effective radial heat conduction of the optimal path, $\kappa_{\text {eff }}$, is defined according to the relation,

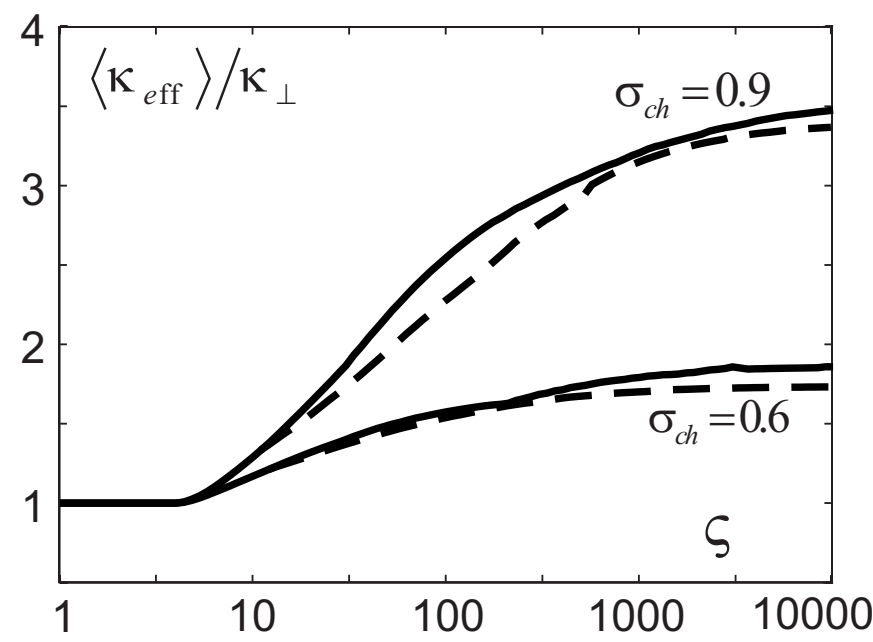

FIG. 4. The ratio of the effective radial heat conduction to the perpendicular one as a function of the parameter $s$ computed for different magnitudes of the Chirikov parameter by taking into account heat paths with up to two (solid curve) and one (dashed curves) parallel sections.

$$
\Gamma_{r}=\kappa_{\mathrm{eff}} \frac{\Delta T}{w y_{0} / 2} .
$$

After averaging over the path initial phase at the symmetry surface we finally get

$$
\frac{\left\langle\kappa_{\mathrm{eff}}\right\rangle}{\kappa_{\perp}}=\int_{0}^{\pi} \frac{d \psi_{1} / \pi}{1-\sum_{j=1}^{j_{\max }}\left(y_{b}^{j}-y_{t}^{j}-\eta_{j} / \varsigma\right) \sigma_{\mathrm{Ch}}}
$$

with the definition $y_{0}=1 / \sigma_{\mathrm{Ch}}$ taken into account.

Thus $\varsigma=4 \kappa_{\|} b_{r}^{2} / \kappa_{\perp}$ and $\sigma_{\mathrm{Ch}}$ are the only parameters which characterize the enhancement of $\kappa_{\text {eff }}$ and $\left\langle\kappa_{\text {eff }}\right\rangle$ over $\kappa_{\perp}$. Figure 2 shows the $s$-dependence of the ratio $\left\langle\kappa_{\text {eff }}\right\rangle / \kappa_{\perp}$ for different magnitudes of $\sigma_{\mathrm{Ch}}$ computed for paths with up to 3 radial and 2 parallel sections. One can see that the parallel transport contributes to the perpendicular one, i.e., optimal paths include fractions along field lines, if $s \geq 4$. The enhancement level saturates when $s$ exceeds $10^{3}-10^{4}$, depending on $\sigma_{\mathrm{Ch}}$. In this case the temperature change in the island approaches zero in correspondence to the "large" island approximation of Ref. 10.

The optimal paths, beginning with different phases at the symmetry plane, found for different strength of perturbations and $\sigma_{\mathrm{Ch}}=0.9$, are shown in Fig. 3. One can see that such paths can be all possible combinations of radial and parallel sections. With increasing perturbation level and approaching the "large" island limit, the parallel sections approach closer and closer to the separatrix. However the final parallel section is always inside the island because (i) at the separatrix the parallel transport becomes less and less efficient by approaching to the X-point and (ii) beyond the island all parallel segments terminate at a finite distance from the RS, i.e., the heat path has to be finished with a radial section contributing strongly to $\Delta T$ for $\mathrm{\gg} \gg 1$.

Finally, in Fig. 4 we compare the transport enhancement obtained by considering paths with maximally one and two 
parallel sections. One can see that the difference between two approaches is not very significant. This is an indication that further increase of the number of parallel sections taken into account, which would lead to a significant rise of computational time, will not noticeably change the results.

This study has been partly performed within GRK1203 of the German Research Society.

${ }^{1}$ Z. Chang, J. D. Callen, E. D. Fredrickson, R. V. Budny, C. C. Hegna, K. M. McGuire, M. C. Zarnstorff, and TFTR group, Phys. Rev. Lett. 74, 4663 (1995).

${ }^{2}$ S. C. McCool, A. J. Wootton, M. Kotschenreuther, A. Y. Aydemir, R. V. Bravenec, J. S. DeGrassie, T. E. Evans, R. L. Hickok, B. Richards, W. L. Rown, and P. M. Schoch, Nucl. Fusion 30, 167 (1990).

${ }^{3}$ H. Tamai, T. Shoji, K. Nagashima, Y. Miura, T. Yamauchi, H. Ogawa, H. Kawashima, T. Matsuda, M. Mori, K. Ida, and S. Ohdachi, J. Nucl. Mater. 220-222, 365 (1995).

${ }^{4} \mathrm{Ph}$. Ghendrih, A. Grosman, and H. Capes, Plasma Phys. Controlled Fusion 38, 1653 (1996)

${ }^{5}$ R. C. Wolf, W. Biel, M. F. M. de Bock, K. H. Finken, S. Günter, G. M. D. Hogeweij, S. Jachmich, M. W. Jakubowski, R. J. E. Jaspers, A. KrämerFlecken, H. R. Koslowski, M. Lehnen, Y. Liang, B. Unterberg, S. K. Varshney, M. von Hellermann, Q. Yu, O. Zimmermann, S. S. Abdullaev, A. J. H. Donné, U. Samm, B. Schweer, M. Tokar, E. Westerhof, and the TEXTOR Team, Nucl. Fusion 45, 1700 (2005).

${ }^{6}$ T. E. Evans, R. A. Moyer, P. R. Thomas, J. G. Watkins, T. H. Osborne,
J. A. Boedo, E. J. Doyle, M. E. Fenstermacher, K. H. Finken, R. J. Groebner, M. Groth, J. H. Harris, R. J. La Haye, C. J. Lasnier, S. Masuzaki, N. Ohyabu, D. G. Pretty, T. L. Rhodes, H. Reimerdes, D. L. Rudakov, M. J. Schaffer, G. Wang, and L. Zeng, Phys. Rev. Lett. 92, 235003 (2004)

${ }^{7}$ T. E. Evans, R. A. Moyer, K. H. Burrell, M. E. Fenstermacher, I. Joseph, A. W. Leonoard, T. H. Osborne, G. D. Portter, M. J. Schaffer, P. B. Snyder, P. R. Thomas, J. G. Watkins, and W. P. West, J. Manuf. Syst. 2, 419 (2006)

${ }^{8}$ Y. Liang, H. R. Koslowski, P. R. Thomas, E. Nardon, B. Alper, P. Andrew, Y. Andrew, G. Arnoux, Y. Baranov, M. Bécoulet, M. Beurskens, T. Biewer, M. Bigi, K. Crombe, E. De La Luna, P. de Vries, W. Fundamenski, S. Gerasimov, C. Giroud, M. P. Gryaznevich, N. Hawkes, S. Hotchin, D. Howell, S. Jachmich, V. G. Kiptily, L. Moreira, V. Parail, S. D. Pinches, E. Rachlew, and O. Zimmermann, Phys. Rev. Lett. 98, 265004 (2007).

${ }^{9}$ M. Z. Tokar, Proceedings of the 14th European Conference on Controlled Fusion and Plasma Physics, Madrid, 1987, edited by F. Engelmann and J. L. Alvarez Rivas (European Physcal Society, Geneva, 1987), Vol. 13F/2, p. 687.

${ }^{10}$ R. Fitzpatrick, Phys. Plasmas 2, 825 (1995).

${ }^{11}$ Q. Yu, Phys. Plasmas 13, 062310 (2006).

${ }^{12}$ M. Holzl, S. Günter, Q. Yu, and K. Lackner, Phys. Plasmas 14, 052501 (2007).

${ }^{13}$ M. Z. Tokar, Phys. Plasmas 6, 2808 (1999).

${ }^{14}$ I. Prigogine, Introduction to Thermodynamics of Irreversible Processes, 2nd ed. (Interscience, New York, 1961), p. 53.

${ }^{15}$ S. I. Braginskii, in Reviews of Plasma Physics edited by M. A. Leontovich (Consultants Bureau, New York, 1965), Vol. 1, p. 205. 